\title{
Screening for proximal coronary artery anomalies with 3-dimensional MR coronary angiography
}

\author{
Niek H. Prakken • Maarten J. Cramer • \\ Marlon A. Olimulder • Pierfrancesco Agostoni • \\ Willem P. Mali • Birgitta K. Velthuis
}

Received: 5 December 2009/Accepted: 8 March 2010/Published online: 26 March 2010

(C) The Author(s) 2010. This article is published with open access at Springerlink.com

\begin{abstract}
Under 35 years of age, 14\% of sudden cardiac death in athletes is caused by a coronary artery anomaly (CAA). Free-breathing 3-dimensional magnetic resonance coronary angiography (3D-MRCA) has the potential to screen for CAA in athletes and non-athletes as an addition to a clinical cardiac MRI protocol. A 360 healthy men and women (207 athletes and 153 non-athletes) aged 18-60 years (mean age $31 \pm 11$ years, $37 \%$ women) underwent standard cardiac MRI with an additional 3D-MRCA within a maximum of 10 min scan time. The 3D-MRCA was screened for CAA. A 335 (93\%) subjects had a technically satisfactory 3D-MRCA of which 4 (1\%) showed a malignant variant of the right coronary artery (RCA) origin running between the aorta and the pulmonary trunk. Additional findings included three subjects with ventral rotation of the RCA with kinking and possible proximal stenosis, one person with additional stenosis and six persons with proximal myocardial bridging of the left anterior descending coronary artery. Coronary CT-angiography (CTA)
\end{abstract}

N. H. Prakken $(\bowtie) \cdot$ W. P. Mali · B. K. Velthuis Department of Radiology, University Medical Center Utrecht, Heidelberglaan 100, 3584 CX Utrecht,

The Netherlands

e-mail: n.prakken@umcutrecht.nl;

nhjprakken@gmail.com

M. J. Cramer · M. A. Olimulder · P. Agostoni Department of Cardiology, University Medical Center Utrecht, Utrecht, The Netherlands was offered to persons with CAA (the CAA was confirmed in three, while one person declined CTA) and stenosis (the ventral rotation of the RCA was confirmed in two but without stenosis, while two people declined CTA). Overall 3D MRCA quality was better in athletes due to lower heart rates resulting in longer end-diastolic resting periods. This also enabled faster scan sequences. A 3D-MRCA can be used as part of the standard cardiac MRI protocol to screen young competitive athletes and non-athletes for anomalous proximal coronary arteries.

Keywords Athletes - Magnetic resonance coronary angiography - Coronary artery anomalies . Coronary artery disease $\cdot$ Myocardial bridging

\section{Introduction}

Coronary artery anomalies (CAA) are the second most common cardiovascular cause (14\%) of sudden cardiac death (SCD) in athletes under 35 years of age, following hypertrophic cardiomyopathy (36\%) [1]. Coronary artery disease (CAD, 3\%) and myocardial bridging $(<3 \%)$ are uncommon causes of SCD in young athletes in contrast to athletes over 35 years where $80 \%$ of SCD is caused by atherosclerotic disease with significant coronary stenosis [2-5].

High risk CAA involve a malignant course between aorta and pulmonary trunk which can be compressed during exercise. A left anterior descending (LAD) 
coronary artery originating from the right sinus is most commonly associated with SCD, followed by the right coronary artery (RCA) originating from the left sinus $[2,6]$.

While multi-detector computed tomography (MDCTA) angiography is faster and more adequate than magnetic resonance angiography (MRA) for the identification of CAD and relevant coronary stenosis, MRA without contrast and radiation is preferable in younger athletes where it suffices to rule out CAA.

Standard cardiac magnetic resonance imaging (MRI) multiple-thin-slab angiography is time-consuming as it requires breath-holding and knowledge of the exact position of the coronary arteries [7], while free-breathing 3-dimensional magnetic resonance coronary angiography (3D-MRCA) only requires the location of the proximal aorta and pulmonary trunk to position the scan volume [8]. 3D-MRCA of the whole heart takes $20 \mathrm{~min}$ on average (range 15-27) leaving limited time for additional imaging of cardiac anatomy and function [8]. As only the proximal coronary arteries are of interest to rule out CAA, a shorter scan with limited coverage can be combined with clinical cardiac anatomy and functional MRI.

The purpose of this study was to test if a $10 \mathrm{~min}$ free-breathing 3D-MRCA scan can be included in the clinical cardiac MRI protocol to rule-out CAA in athletes and non-athletes.

\section{Methods}

Study population

For this study 360 men and women, aged 18-60 years (mean age $31 \pm 11$ years, $37 \%$ women), were selected from a larger study of 480 healthy volunteers who were prospectively recruited over 1.5 years with the aim of acquiring normal cardiac MRI reference values in healthy athletes and non-athletes. The only selection criteria for the 360 subjects was that there was sufficient remaining scan time to perform the freebreathing 3D-MRCA additional to the standard cardiac anatomic and functional MRI performed in all 480 volunteers $[9,10]$.

Before participating in the larger study, all persons were interviewed, completed a questionnaire regarding training history, past medical and family history, and underwent resting electrocardiography and blood pressure measurement before cardiac MRI. Persons could only participate in the larger study if there was no history of illness, hypertension or medication. Persons with hypertension, unexpected findings on the electrocardiogram or cardiac MRI during the study, or contraindications for MRI, were excluded. Electrocardiograms were read by a cardiologist according to the European Society of Cardiology pre-participation guidelines [11]. No one who underwent the additional 3D-MRCA was excluded from this study.

There were 207 athletes (exercising at least $9 \mathrm{~h}$ per week), and 153 healthy non-athletes (exercising $\leq 3 \mathrm{~h}$ per week). The Institutional Ethics Committee of the University Medical Center Utrecht approved the study and all persons gave written informed consent.

\section{Acquisition protocol}

All scans were performed on a 1.5-T MRI system (Achieva release 1.5, Philips Medical Systems, Best, The Netherlands). All images were acquired by the same operator (N. H. P) using vector-electrocardiogram triggering with a five-element phased-array cardiac coil for signal reception. The standard protocol, of which the results are described separately, included breathhold steady-state-free-precession cines of different views of both ventricles and flow measurements of the cardiac valves [12].

In addition, a free-breathing steady-state-free-precession 3D-MRCA balanced turbo-field-echo sequence containing the proximal course of the coronary arteries was planned on a coronal view, starting from around $2 \mathrm{~cm}$ above the aortic root to about $5 \mathrm{~cm}$ below the aortic root [13]. The 3D-MRCA was performed in cartesian acquisition mode in the transversal plane including a $\mathrm{T} 2$ preparation pulse and a fat suppression preparation pulse, repetition/echo time 5.4/2.6, flip angle $90^{\circ}$, rectangular field-of-view (FOV) $100 \%$, phase resolution $100 \%$ and sensitivity encoding reduction of two in the anterior-posterior, and one in the feethead direction. Turbo-field-echo factors were maximized individually to match the end-diastolic resting period obtained by visually identifying the time window of least RCA movement during diastole (resting period) on a four-chamber cine [8]. A respiratory navigator was used by placing the 5-mm $2 \mathrm{D}$ selective excitation pulse on the highest point of the liver on the right side of the diaphragm. To optimize scan-time while maintaining a reconstructed isotropic 
in-plane resolution of $0.55 \mathrm{~mm}^{2}$, the FOV was minimized and matrix size was adjusted without introducing fold-over artifacts per scan. Typically the matrix was reconstructed to obtain a twofold higher resolution in two directions after reconstruction; a matrix of $256 \times 256$ was reconstructed to $512 \times 512$, so that the scanned in-plane spatial resolution of $1.1 \mathrm{~mm}^{2}$ was reconstructed to an in-plane resolution of $0.55 \mathrm{~mm}^{2}$. We planned the nominal scan duration under 4 min so that the effective scan duration would not exceed 10 min with a minimum navigator efficiency of $40 \%$. Exact nominal and effective scan duration and navigator efficiency were not recorded. In this pragmatic approach, if the heart rate was less than 60 beats per minute (bpm), we chose the 139 slice package with $0.55 \mathrm{~mm}$ slice thickness. If the scan duration prognosis exceeded the 10 min maximum (including navigator efficiency below $40 \%$ or heart rate above $60 \mathrm{bpm}$ ), the slice thickness and the number of slices were adjusted from 139 slices of $0.55 \mathrm{~mm}$ (isotropic voxels of $0.55 \mathrm{~mm}^{3}$ after reconstruction) to 70 slices of $1 \mathrm{~mm}$ (anisotropic voxels), or if necessary to 35 slices of $2 \mathrm{~mm}$ (anisotropic voxels). No medication was given to lower the heart rate.

Post processing and data analysis

The 3D-MRCA dataset was transferred to a Vitrea workstation version 4.0 (Vital Images) for source image analysis, slab maximum-intensity-projection, and 3-dimensional reconstructions.

A radiologist experienced in cardiac MRI (B. K. V.), and blinded for participant characteristics, scored all 3D datasets on the source images. The overall image quality of the 3D-MRCA was visually graded for CAA; 0 -indicating uninterpretable poor quality (technically unsatisfactory scan/ non diagnostic); 1-moderate (suboptimal visualization of all the major coronary arteries but assessment is still possible); 2-good visibility of all the proximal coronary arteries. The three coronary arteries were also assessed separately using above-mentioned grading system. The anatomic position of the three major coronary arteries and their proximal course in relation to the aortic root and the pulmonary trunk, the presence of proximal lumen reduction of more than $50 \%$, and the presence of myocardial bridging were evaluated.

Final diagnosis was determined for positive findings in a consensus meeting with a cardiologist experienced in cardiac MRI (M. J. C.). Subjects with a CAA or proximal stenosis on 3D-MRCA were offered a low-dose (1-3 mSv) prospectively triggered multidetector CT angiography (MDCTA), and if necessary additional testing, for confirmation. Participants with myocardial bridging were not offered MDCTA as it is a common incidental finding on CT and only very rarely associated with sudden cardiac death in asymptomatic people [4, 6, 14]. The 3DMRCA diagnosis was not systematically confirmed in all subjects as MDCTA was not mandatory because of the required radiation exposure in a perceived healthy population.

Statistical analysis

Continuous data are distributed normally and are presented as mean values \pm standard deviation (SD). $P$-values $<0.05$ were considered statistically significant. Differences between groups were assessed using ANOVA with Bonferroni correction. Categorical data were expressed as frequencies and percentages.

\section{Results}

Study population demographics are shown in Table 1. Athletes had a significantly lower heart rate as a consequence of their significantly higher training intensity. Overall grading of the coronary arteries for assessment of CAA and per-vessel grading results are presented in Table 2. A technically satisfactory (graded moderate or higher) 3D-MRCA was obtained in 335 of 360 scanned subjects (93\%) and 288 of 360 were graded as good ( $80 \%)$. In athletes, $83 \%$ of the 3DMRCA scans were graded as good for CAA assessment, as compared to respectively $75 \%$ in non-athletes. The per-vessel assessment in athletes graded was good in $73 \%$ of the RCA, $66 \%$ of the LAD, and $67 \%$ of the left circumflex (LCX) coronary artery as compared to respectively 67,59 and $56 \%$ in non-athletes.

The number of slices per 3D-MRCA package were 139 slices in $100(28 \%)$ cases; 36/153 (24\%) nonathletes and 64/207 (31\%) athletes, 70 slices in 220 $(61 \%)$, and 35 slices in $40(11 \%)$. A lower heart rate, as seen in athletes, improved coronary anatomy and stenosis assessment significantly (mean heart rate in scans that scored moderate and good were respectively $69 \pm 11$ and $59 \pm 10$ beats per minute, $P<0.0005$ ), 
Table 1 Baseline demographics

\begin{tabular}{lll}
\hline & Non-athletes & All athletes \\
\hline$n$ & 153 & 207 \\
Female & $46 \%$ & $30 \%$ \\
Age (years) & $29 \pm 9.7$ & $32 \pm 12^{*}$ \\
Height (cm) & $178 \pm 9.7$ & $182 \pm 10^{*}$ \\
Weight (kg) & $72 \pm 13$ & $74 \pm 10$ \\
BSA (m $\left.{ }^{2}\right)$ & $1.9 \pm 0.2$ & $1.9 \pm 0.2$ \\
Systolic BP (mmHg) & $127 \pm 16$ & $129 \pm 15$ \\
Diastolic BP (mmHg) & $75 \pm 10$ & $75 \pm 10$ \\
Mean heart rate (bpm) & $65 \pm 11$ & $57 \pm 10^{\ddagger}$ \\
Training intensity (h/week) & $2.0 \pm 1.3$ & $15 \pm 5.9^{\ddagger}$ \\
Smoking (cigarettes/week) & $3.3 \pm 16$ & $0.0 \pm 0.1^{\dagger}$ \\
\hline
\end{tabular}

Data are expressed as mean \pm SD. BSA body surface area, BP blood pressure, $b p m$ beats per minute, $h / w k$ hours endurance exercise training per week

${ }^{a} P$-values for significance in differences between controls and regular endurance athletes

* $P<0.05 ;^{\dagger} P<0.005 ;{ }^{\star} P<0.0005$

Table 2 Grading percentages of general and per-vessel coronary artery assessment for the presence of CAA and significant stenosis

\begin{tabular}{lllll}
\hline & & Poor $(\%)$ & Moderate $(\%)$ & Good (\%) \\
\hline CAA & Non-athletes & 9 & 16 & 75 \\
& Athletes & 7 & 10 & 83 \\
RCA & Non-athletes & 18 & 15 & 67 \\
& Athletes & 12 & 15 & 73 \\
LAD & Non-athletes & 19 & 22 & 59 \\
& Athletes & 13 & 21 & 66 \\
LCX & Non-athletes & 25 & 19 & 56 \\
& Athletes & 15 & 17 & 67 \\
\hline
\end{tabular}

$C A A$ coronary artery anomaly, $R C A$ right coronary artery, $L A D$ left descending coronary artery, $L C X$ left circumflex artery

and facilitated completion of more and thinner slices per package within 10 min scan time.

However, the image grade only had a statistically significant relation to the number of slices per package in the 139-slice package $(P<0.05)$; the 139 -slice package was graded as good in $85 \%, 70$ slices in $78 \%$ and 35 slices in $78 \%$. Coronary segments that were covered by the 3D-MRCA package included the left main coronary artery (LMCA) and the proximal and mid portion of the
LAD (including first diagonal branch), the LCX, and the RCA.

The occurrence of CAA, myocardial bridging and stenosis is presented in Table 3. The radiologist (B. K. V.) found four cases (1\%) with CAA, 4 cases of proximal significant stenosis, and seven cases of proximal myocardial bridging of the LAD. Agreement was reached in all positive cases by consensus reading with the cardiologist (M. J. C). The four CAA cases all showed the RCA running between the aortic root and the pulmonary trunk, three of which originated from the left sinus and one originated higher from the ventral aorta. No LAD originated from the right sinus and there were no LCX anomalies. One of the four cases of stenosis was a LAD stenosis. The other three cases of stenosis concerned a ventral rotation of the RCA originating from the right coronary sinus with a proximal kink, and possible stenosis. RCA ventral rotation of the origin, towards the space between the outflow tract of the right pulmonary artery and aorta, was found significantly more $(P<0.05)$ in athletes [50 subjects (24\%): 26 men, 24 women] than in non-athletes [24 subjects (16\%): 11 men, 13 women].

All cases with CAA and/or possible presence of stenosis were offered a MDCTA for confirmation. Individual imaging results are presented in Figs. 1, 2, 3, 4. Athletes were offered a super Bruce tread-mill protocol until physical exhaustion [15] and nonathletes a standard Bruce tread-mill protocol, which if positive was followed by X-ray coronary angiography and treatment if necessary.

\section{Discussion}

Our results show that the origin and the proximal course of the coronary arteries are well depicted with 3D-MRCA, but the visualization of stenoses is less reliable. A 10 min 3D-MRCA protocol can be used to screen for CAA. This MRCA protocol was designed for the detection of coronary anomalies and not for coronary stenosis detection.

\section{Comparison to the literature}

The number of interpretable 3D-MRCA scans is comparable to the $84 \%$ reported by Kim [16]. Of all 335 technically satisfactory scans, the RCA originated 
Table 3 Coronary artery anomalies and additional findings (stenoses and myocardial bridging)

\begin{tabular}{|c|c|c|c|c|c|c|}
\hline Gender & Age & Athlete & 3D-MRCA & $\begin{array}{l}\text { Malignant } \\
\text { CAA }\end{array}$ & $\begin{array}{l}\text { Invitation } \\
\text { MDCTA }\end{array}$ & Result MDCTA \\
\hline Man & 28 & Yes & Left sinus RCA origin, bridging mid-LAD & Yes & Yes & $\begin{array}{l}\text { Left sinus RCA origin, } \\
\text { LAD bridging (Fig. 1) }\end{array}$ \\
\hline Man & 46 & Yes & Left sinus RCA origin & Yes & Yes & $\begin{array}{l}\text { Left sinus RCA origin, } \\
\text { Mid-RCA stenosis (Fig. 3) }\end{array}$ \\
\hline Woman & 31 & Yes & Left sinus RCA origin & Yes & Yes & Declined invitation \\
\hline Man & 21 & No & High ventral aortic RCA origin & Yes & Yes & $\begin{array}{l}\text { Left sinus RCA origin } \\
\text { (Fig. 2) }\end{array}$ \\
\hline Man & 35 & Yes & \multirow{3}{*}{$\begin{array}{l}\text { Ventral rotation of RCA, proximal kinking/ } \\
\text { stenosis }\end{array}$} & No & Yes & \multirow{2}{*}{$\begin{array}{l}\text { RCA rotation, no stenosis } \\
\text { (Fig. 4) }\end{array}$} \\
\hline Man & 55 & Yes & & No & Yes & \\
\hline Man & 21 & Yes & & No & Yes & Declined invitation \\
\hline Man & 33 & Yes & Mid-LAD stenosis & No & Yes & Declined invitation \\
\hline Woman & 29 & Yes & Mid-LAD bridging & No & No & - \\
\hline Man & 32 & Yes & & No & No & - \\
\hline Man & 29 & Yes & First diagonal branch bridging & No & No & - \\
\hline Man & 53 & Yes & & No & No & - \\
\hline Man & 21 & Yes & & No & No & - \\
\hline Woman & 48 & No & & No & No & - \\
\hline
\end{tabular}

3D-MRCA 3-dimensional MR coronary angiography, $C A A$ coronary artery anomaly, MDCTA multidetector computed tomographic angiography, stenosis proximal coronary artery lumen reduction $>50 \%, R C A$ right coronary artery, $L A D$ left descending coronary artery

from the left sinus in three cases $(0.90 \%)$, one RCA originated higher from the ventral aorta, and no LAD originated from the right sinus, which is comparable to the $\mathrm{x}$-ray coronary angiography prevalence as reported by Angelini; $0.92 \%$ for RCA and $0.15 \%$ for LAD. [5, 17] The MDCTA incidence of CAA is $2.5 \%$ as reported by Schmitt including the RCA originating outside its sinus in $0.40 \%$ and the left coronary arteries (both LAD and LCA) in $0.74 \%$ (a LAD originating outside its sinus with a normal LCA was not observed in their study)[18]. Although occurring infrequently, physical exercise is suggested to be highly related to SCD when the LAD has an anomalous origin from the right sinus and runs between the aortic root and the pulmonary trunk $[6,19]$. One study reports that an anomalous origin of the RCA from the left sinus, as found in three of our subjects, occasionally gives major symptoms such as chest-pain or syncope and is the cause of $13 \%$ of CAA related SCD [2]. An unusually high anterior origin of the RCA from the ascending aorta ("high take-off position") and continuing down at a steep angle to the aorta as seen in one of our male non-athletes, has been described earlier by Schmitt and found in two $(0.11 \%)$ of 1758 MDCTA screened patients suspected for CAD [18].

Myocardial bridging of the LAD was observed in seven of our cases, which was only confirmed in one person by MDCTA as the other subjects did not undergo MDCTA. The actual prevalence of asymptomatic myocardial bridging varies widely between x-ray coronary angiographic studies (0.5-2.5\%) [20, 21], MDCTA (17-58\%) [4, 14, 22] and autopsy series (15-85\%), and most commonly affects the LAD [4, 21]. Recent MDCTA results are comparable to autopsy series which is the gold standard for detecting myocardial bridging [23].

Origin rotation variation of the RCA and the LCA with normal coronary origin from the sinus of Valsalva, caused by rotation of the aortic root between 45 and $90^{\circ}$, have been described earlier although only found in $0.22 \%$.[18].

\section{Clinical implications}

A 10 min 3D-MRCA protocol can be used as a non-invasive screening tool to exclude CAA in asymptomatic athletes under 35 years of age as they have low risk of 


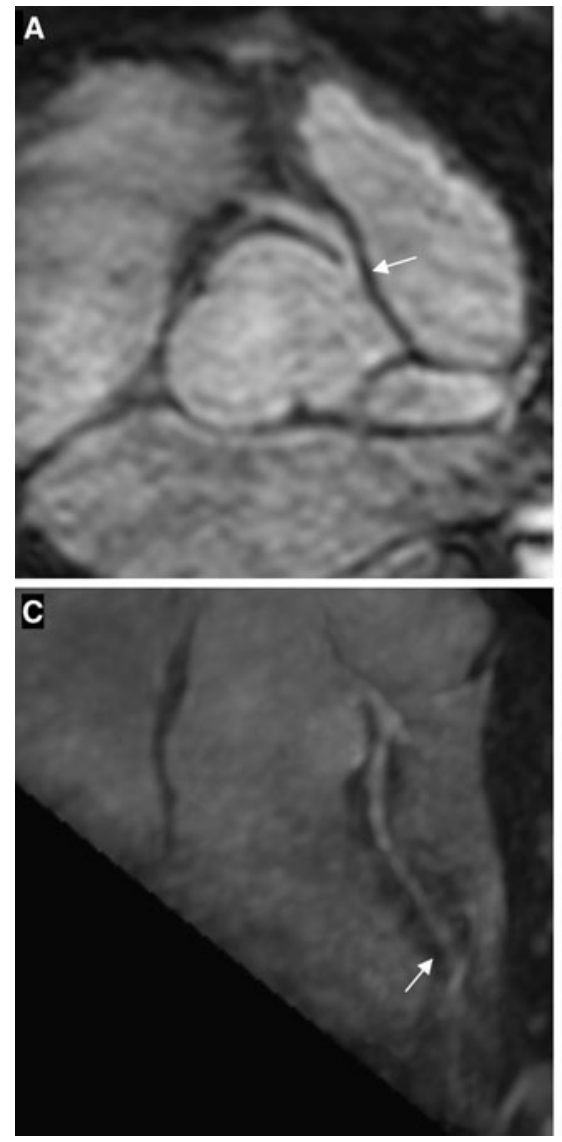

Fig. 1 A 28 year old male athlete with a malignant course of the RCA between the aorta and the pulmonary trunk with slight narrowing of the RCA origin on 3D-MRCA (a) and MDCTA (b). Bridging (intramuscular course) of the mid-LAD on 3D-
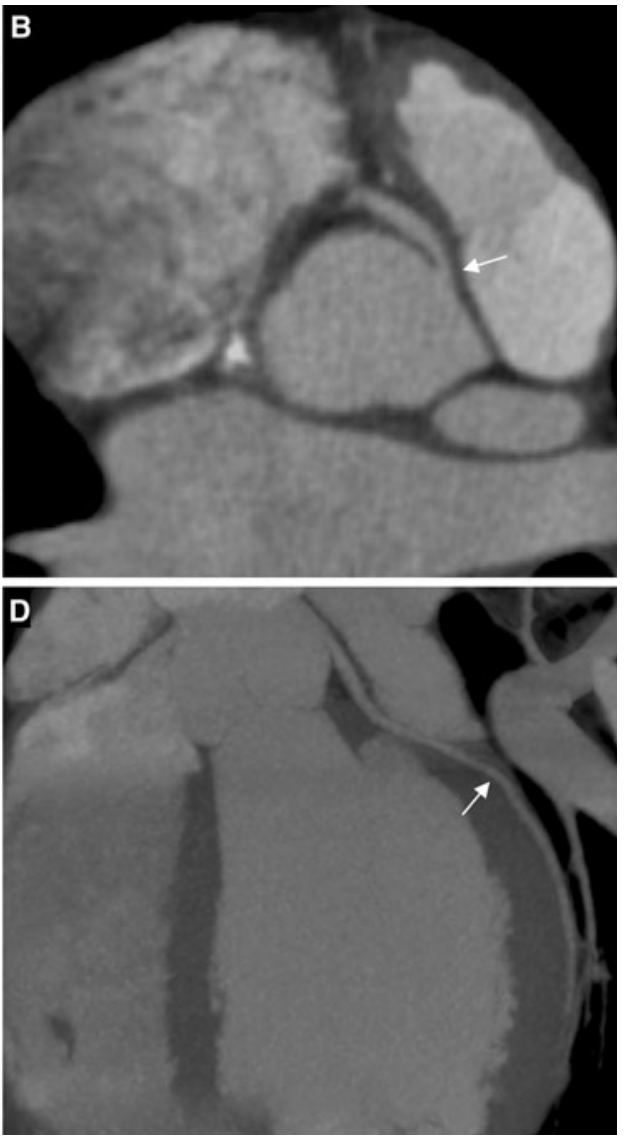

MRCA (c) and MDCTA (d). The super Bruce tread-mill test and scintigraphy were negative. No X-ray coronary angiography was performed

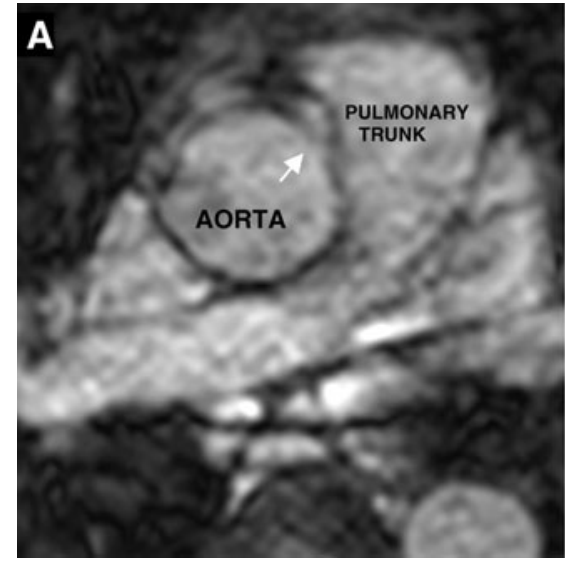

Fig. 2 A 21 year old male non-athlete with a high ventral aorta origin RCA on 3D-MRCA (a: axial image, b: coronal oblique image) and confirmed on MDCTA (c). The standard
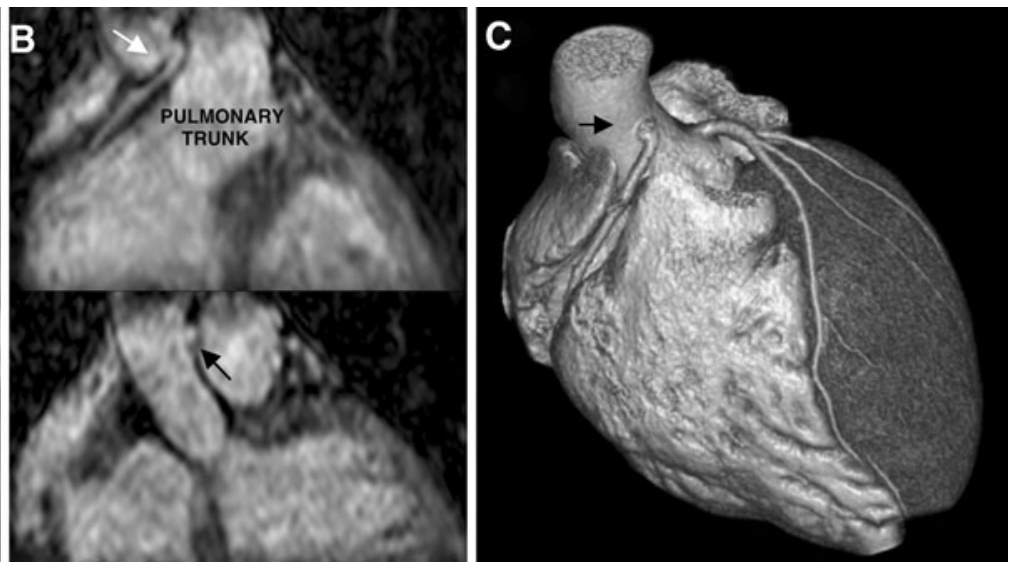

Bruce tread-mill test was negative. No scintigraphy or X-ray coronary angiography was performed 

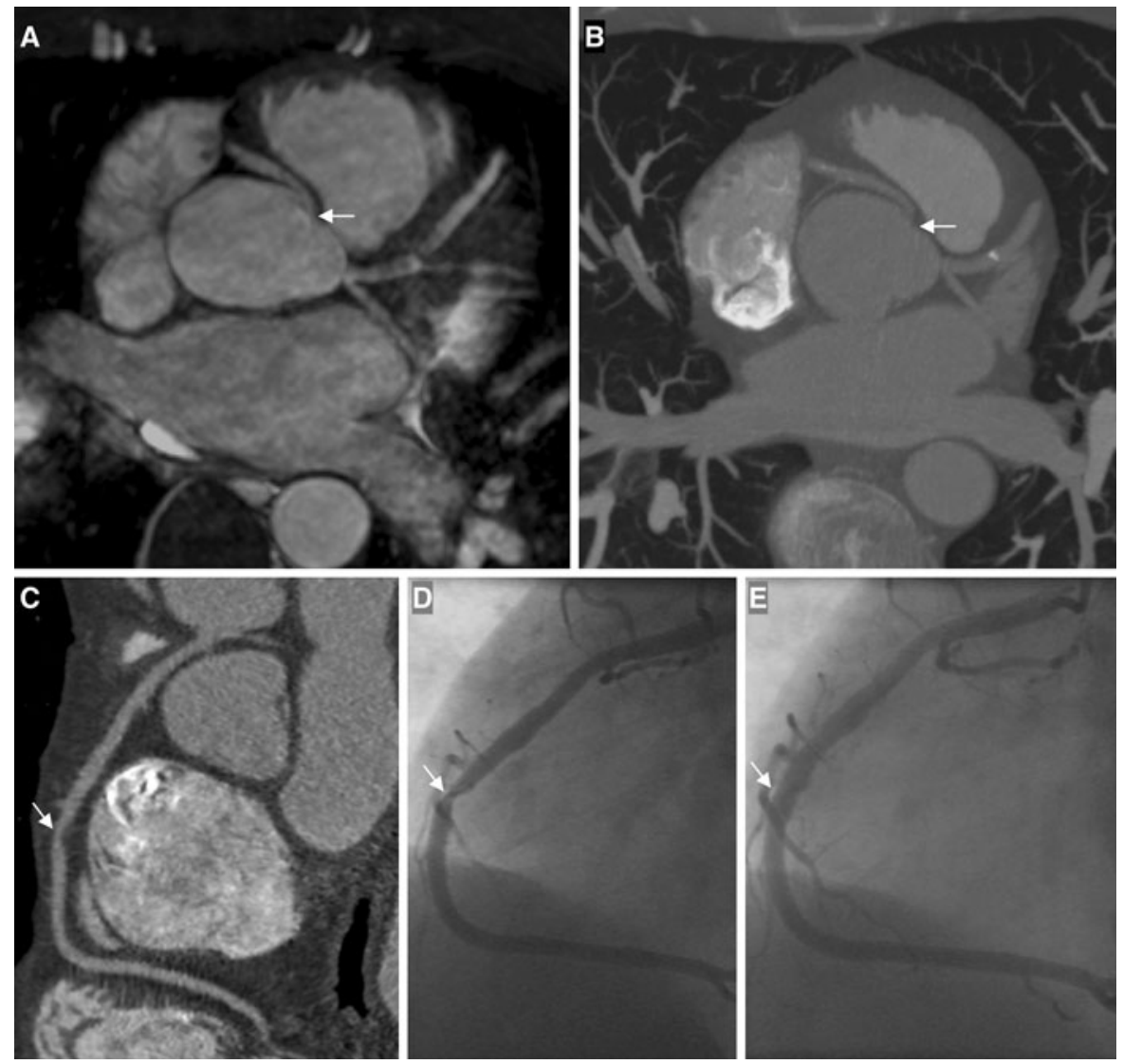

Fig. 3 A 46 year old male athlete with left sinus origin and malignant course of the RCA between the aorta and pulmonary trunk on 3D-MRCA (a) and MDCTA (b). An additional significant soft-plaque stenosis of the mid-RCA (70\% lumen narrowing) on MDCTA (c) was missed on 3D-MRCA due to limited coverage. The Agatston coronary calcium score was negative. The super Bruce treadmill test was positive. No scintigraphy was performed. An X-ray coronary angiogram showed an $80 \%$ lumen stenosis of the mid-RCA (d). Percutaneous coronary intervention of mid-RCA with bare metal stent implantation was performed (e)

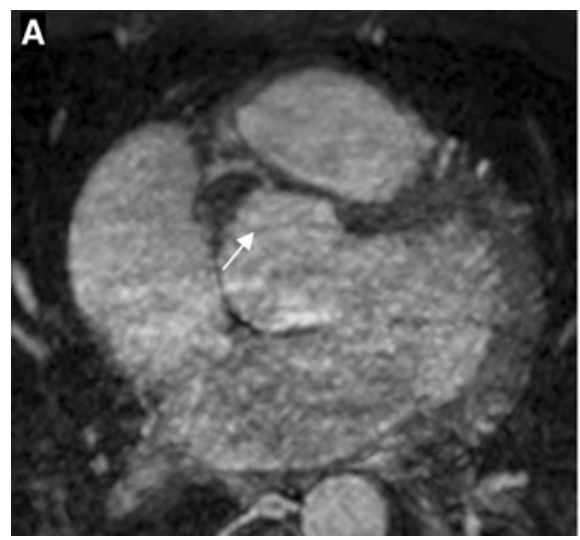

Fig. 4 A 35 year old male athlete with a ventral rotation of RCA originating from the right coronary sinus without malignant course but with possible stenosis on 3D-MRCA (a). The ventral rotation origin of the RCA was confirmed, but

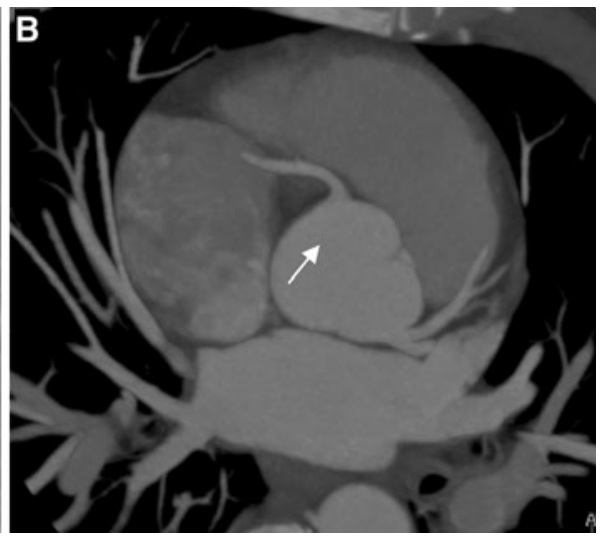

no stenosis was seen on MDCTA (b). The super Bruce treadmill test was negative. No scintigraphy or X-ray coronary angiography was performed 
CAD [3, 24]. This MRCA protocol, with limited resolution and coverage within the time-limit, was designed for the detection of coronary anomalies and not for coronary stenosis detection. Consequently, older athletes and athletes with cardiovascular complaints should either undergo dedicated full-coverage 3D-MRCA or prospectively triggered MDCTA [24]. Figure 3 illustrates the limited coverage shortcoming of this 3D-MRCA protocol: a soft plaque with a severe stenosis was found in the mid-segment of the RCA on MDCTA and this segment was outside the coverage of the 3D-MRCA. A low dose prospectively triggered MDCTA provides high resolution images which can confirm CAA and visualize the extent of associated intramural course and lumen stenosis, and rule out additional stenosis as it covers the entire coronary artery tree.

Conventional exercise stress testing can be an insufficient stress in a well-trained athlete. Additional testing in an athlete with CAA and/or asymptomatic stenosis can involve exercise testing until physical exhaustion using the super Bruce tread-mill protocol instead of the standard Bruce tread-mill protocol, to detect local ischemia or arrhythmias [25]. If positive, a x-ray coronary angiography or scintigraphy can be performed.

Myocardial bridges in the LAD are a common finding with intramyocardial segments often showing mild-to-moderate luminal narrowing at rest, which is higher during end-systolic phase, but rarely causes angina or infarction through intermittent phase-dependent vessel compression [4]. As a prospective MDCTA is usually only acquired in the end-diastolic phase of the cardiac cycle it will not demonstrate possible narrowing during systole [1]. If detected, myocardial bridging in symptomatic patients can be treated with $\beta$ blockers, nitrates or calcium antagonists and, in extremely selected cases, percutaneous coronary intervention or coronary artery bypass grafting [4].

\section{Limitations}

Although $93 \%$ of the 3D-MRCA scans were technically satisfactory, $\beta$-blockade could further improve image quality as it lowers heart-rate and heart rate variability and increases the end-diastolic resting period significantly [26]. However, little improvement may be expected in athletes with a regular heart rate below 60 beats per minute.
The main limitation of this study is the lack of validation with MDCTA or X-ray coronary angiography in subjects without a coronary artery anomaly. This study was not set up as a side-by-side comparison to MDCTA or X-ray coronary angiography as previous studies focusing on these comparisons have been already performed and the amount of radiation exposure was considered unethical in these healthy subjects $[27,28]$. We discussed the results individually and left the people the choice for further diagnostic testing. As a consequence, test characteristics such as sensitivity and specificity cannot be presented. This study focuses only on the detection of proximal CAA in asymptomatic subjects.

Future developments

1.5 Tesla MR scanners using five-element phasedarray cardiac-coils are the current standard for cardiac imaging. SNR advantages of 3.0 Tesla can be used to improve coronary artery image resolution and shorten the scan time, but 3.0 Tesla is currently hampered by increased susceptibility artifacts. Future optimization of 3.0 Tesla techniques will increase clinical use [29]. Increasing numbers of channels in multiple-channel coils can improve SNR and parallel imaging performance, however, reconstruction time and increased computer memory requirement become inevitable problems which need to be solved by advanced software still in experimental stage [30].

\section{Conclusion}

3D-MRCA can depict coronary origin and the proximal course well. Lower heart rates greatly improved overall image quality in athletes. This 10 min 3D-MRCA protocol can be used to screen for proximal CAA in young asymptomatic athletes and non-athletes, however, it is not suitable for the detection of coronary stenosis.

\section{Acknowledgments None.}

Open Access This article is distributed under the terms of the Creative Commons Attribution Noncommercial License which permits any noncommercial use, distribution, and reproduction in any medium, provided the original author(s) and source are credited. 


\section{References}

1. Prakken NH, Velthuis BK, Cramer MJ, Mosterd A (2009) Advances in cardiac imaging: the role of magnetic resonance imaging and computed tomography in identifying athletes at risk. Br J Sports Med 43:677-684

2. Maron BJ, Doerer JJ, Haas TS, Tierney DM, Mueller FO (2009) Sudden deaths in young competitive athletes: analysis of 1866 deaths in the United States, 1980-2006. Circulation 119:1085-1092

3. Maron BJ, Thompson PD, Ackerman MJ et al (2007) Recommendations and considerations related to preparticipation screening for cardiovascular abnormalities in competitive athletes: 2007 update: a scientific statement from the American Heart Association Council on Nutrition, Physical Activity, and Metabolism: endorsed by the American College of Cardiology Foundation. Circulation 115:1455-1643

4. Jodocy D, Aglan I, Friedrich G et al (2010) Left anterior descending coronary artery myocardial bridging by multislice computed tomography: correlation with clinical findings. Eur J Radiol 73:89-95

5. Angelini P, Velasco JA, Flamm S (2002) Coronary anomalies: incidence, pathophysiology, and clinical relevance. Circulation 105:2449-2454

6. Angelini P (2007) Coronary artery anomalies: an entity in search of an identity. Circulation 115:1296-1305

7. McConnell MV, Stuber M, Manning WJ (2000) Clinical role of coronary magnetic resonance angiography in the diagnosis of anomalous coronary arteries. J Cardiovasc Magn Reson 2:217-224

8. Prakken NH, Vonken EJ, Velthuis BK, Doevendans PA, Cramer MJ (2006) 3D MR coronary angiography: optimization of the technique and preliminary results. Int $\mathrm{J}$ Cardiovasc Imaging 22:477-487

9. Prakken NH, Velthuis BK, Teske AJ, Mosterd A, Mali WP, Cramer MJ (2009) Cardiac MRI reference values for athletes and nonathletes corrected for body surface area, training hours/week and sex. Eur J Cardiovasc Prev Rehabil (Epub ahead of print)

10. Prakken NH, Cramer MJ, Teske AJ, Arend M, Mali WP, Velthuis BK (2010) The effect of age in the cardiac MRI evaluation of the athlete's heart. Int J Cardiol (Epub ahead of print)

11. Corrado D, Pelliccia A, Bjornstad HH et al (2005) Cardiovascular pre-participation screening of young competitive athletes for prevention of sudden death: proposal for a common European protocol. consensus statement of the study group of sport cardiology of the working group of cardiac rehabilitation and exercise physiology and the working group of myocardial and pericardial diseases of the European society of cardiology. Eur Heart J 26: 516-524

12. Prakken NH, Velthuis BK, Bosker AC et al (2009) Relationship of ventricular and atrial dilatation to valvular function in endurance athletes. Br J Sports Med (Epub ahead of print)

13. Weber OM, Martin AJ, Higgins CB (2003) Whole-heart steady-state free precession coronary artery magnetic resonance angiography. Magn Reson Med 50:1223-1228
14. Kim PJ, Hur G, Kim SY et al (2009) Frequency of myocardial bridges and dynamic compression of epicardial coronary arteries: a comparison between computed tomography and invasive coronary angiography. Circulation 119:1408-1416

15. Oury JH, Doty DB, Oswalt JD, Knapp JF, Mackey SK, Duran CM (1998) Cardiopulmonary response to maximal exercise in young athletes following the ross procedure. Ann Thorac Surg 66:S153-S154

16. Kim WY, Danias PG, Stuber M et al (2001) Coronary magnetic resonance angiography for the detection of coronary stenoses. N Engl J Med 345:1863-1869

17. Davis JA, Cecchin F, Jones TK, Portman MA (2001) Major coronary artery anomalies in a pediatric population: incidence and clinical importance. J Am Coll Cardiol 37:593-597

18. Schmitt R, Froehner S, Brunn J et al (2005) Congenital anomalies of the coronary arteries: imaging with contrastenhanced, multidetector computed tomography. Eur Radiol 15:1110-1121

19. Basso C, Maron BJ, Corrado D, Thiene G (2000) Clinical profile of congenital coronary artery anomalies with origin from the wrong aortic sinus leading to sudden death in young competitive athletes. J Am Coll Cardiol 35:1493-1501

20. Tsujita K, Maehara A, Mintz GS et al (2008) Comparison of angiographic and intravascular ultrasonic detection of myocardial bridging of the left anterior descending coronary artery. Am J Cardiol 102:1608-1613

21. Mohlenkamp S, Hort W, Ge J, Erbel R (2002) Update on myocardial bridging. Circulation 106:2616-2622

22. Atar E, Kornowski R, Fuchs S, Naftali N, Belenky A, Bachar GN (2007) Prevalence of myocardial bridging detected with 64-slice multidetector coronary computed tomography angiography in asymptomatic adults. J Cardiovasc Comput Tomogr 1:78-83

23. Lazoura O, Kanavou T, Vassiou K, Gkiokas S, Fezoulidis IV (2010) Myocardial bridging evaluated with 128-multi detector computed tomography coronary angiography. Surg Radiol Anat 32:45-50

24. Pouleur AC, Waroux JB, Kefer J, Pasquet A, Vanoverschelde JL, Gerber BL (2008) Direct comparison of wholeheart navigator-gated magnetic resonance coronary angiography and 40- and 64-slice multidetector row computed tomography to detect the coronary artery stenosis in patients scheduled for conventional coronary angiography. Circ Cardiovasc Imaging 1:114-121

25. Grewal J, McCully RB, Kane GC, Lam C, Pellikka PA (2009) Left ventricular function and exercise capacity. JAMA 301:286-294

26. Jahnke C, Paetsch I, Achenbach S et al (2006) Coronary MR imaging: breath-hold capability and patterns, coronary artery rest periods, and beta-blocker use. Radiology 239:71-78

27. Liu X, Zhao X, Huang J et al (2007) Comparison of 3D freebreathing coronary MR angiography and 64-MDCT angiography for detection of coronary stenosis in patients with high calcium scores. AJR Am J Roentgenol 189:1326-1332

28. Danias PG, Roussakis A, Ioannidis JP (2004) Diagnostic performance of coronary magnetic resonance angiography as compared against conventional X-ray angiography: a meta-analysis. J Am Coll Cardiol 44:1867-1876 
29. Yang PC, Nguyen P, Shimakawa A et al (2004) Spiral magnetic resonance coronary angiography-direct comparison of 1.5 Tesla vs. 3 Tesla. J Cardiovasc Magn Reson 6:877-884
30. Huang F, Vijayakumar S, Akao J (2005) Software compression for partially parallel imaging with multi-channels. Conf Proc IEEE Eng Med Biol Soc 2:1348-1351 